\title{
Diversity Analysis for Frequency-Selective MIMO-OFDM Systems With General Spatial and Temporal Correlation Model
}

\author{
Ahmed K. Sadek, Student Member, IEEE, Weifeng Su, Member, IEEE, and K. J. Ray Liu, Fellow, IEEE
}

\begin{abstract}
In this paper, the effect of a general spatial and temporal fading correlation structure on the performance of coded multiple-input multiple-output (MIMO)-orthogonal frequency-division multiplexing (OFDM) systems is studied. The analysis handles an arbitrary joint transmit-receive spatial correlation model, including the non-Kronecker model. An upper bound on the maximum achievable diversity order for frequency-selective MIMO-OFDM systems with general temporal and spatial correlation is derived. Furthermore, a space-time-frequency code design that can achieve the upper bound for any arbitrarily correlated channel scenario is provided. The general framework of the analysis includes space-frequency (SF)-coded systems as a special case. For the SF-coded MIMO-OFDM system, it is shown that any SF code designed to achieve full diversity in the independent fading channel can achieve full diversity in an arbitrary spatially correlated channel. The derived analytical results are consistent with those in the existing literature for special correlation structures. Extensive simulation results are provided to confirm the theoretical analysis.
\end{abstract}

Index Terms-Broadband wireless communications, maximum achievable diversity, multiple antennas, multiple-input multiple-output (MIMO)-orthogonal frequency-division multiplexing (OFDM) systems, non-Kronecker model, space-frequency (SF) coding, space-time-frequency (STF) coding.

\section{INTRODUCTION}

$\mathbf{O}$ NE OF THE major challenges that designers of wireless communication systems face is reliably conveying information to the receiver in the presence of signal fading. To achieve this goal, various diversity schemes can be used. The diversity order of a system can be defined as the number of independent channels over which information is being sent; it also denotes the degrees of freedom (DOFs) associated with the system. There are three physical domains in which we can generate independent channels: time, frequency, and space. The use of spatial diversity, especially transmit diversity, has

Paper approved by J. Wang, the Editor for Wireless Spread Spectrum of the IEEE Communications Society. Manuscript received October 12, 2004; revised April 24, 2005 and August 9, 2005. This work was supported in part by the Army Research Laboratory under Grant DAAD 190120011. This paper was presented in part at the IEEE Global Telecommunications Conference (GLOBECOM), Dallas, TX, December 2004.

A. K. Sadek and K. J. R. Liu are with the Department of Electrical and Computer Engineering, and Institute for Systems Research, University of Maryland, College Park, MD 20742 USA (e-mail: aksadek@eng.umd.edu; kjrliu@eng. umd.edu).

W. Su is with the Department of Electrical Engineering, State University of New York at Buffalo, Buffalo, NY 14260 USA (e-mail: weifeng@eng.buffalo. edu).

Digital Object Identifier 10.1109/TCOMM.2006.873986 gained a lot of interest in the recent years, as it does not incur any loss in spectral efficiency in the system. Previous work on transmit diversity assumed independence of the spatial fading across different transmit and receive antenna elements. However, in practical scenarios, this assumption is not always valid, because of insufficient antenna spacing and/or lack of scatterers. Most of the work that studied the effect of correlation on the performance of multiple-input multiple-output (MIMO) systems considered a narrowband flat-fading channel [1]-[4], where space-time (ST) coding is usually used.

For the more interesting case of frequency-selective fading channels, which generally arises in broadband wireless systems, little work has studied the effect of spatial correlation on the performance of the system. In addition to the spatial diversity inherent in the MIMO broadband system, frequency diversity is also offered by the multipath nature of the channel. Due to the frequency-selective nature of the broadband channel, orthogonal frequency-division multiplexing (OFDM) is an attractive approach to reduce the complexity of equalization and decoding [5], [6]. To exploit both the spatial and frequency diversities, space-frequency (SF) coding is used over each OFDM block. The authors in [7] and [8] have proposed systematic SF code-design methodologies that guarantee achieving full spatial and frequency diversities. However, these code designs assumed full independence among the spatial fading of different antenna element pairs. In [9], a space-time-frequency (STF) code was presented to achieve the spatial and frequency diversity without any temporal diversity gain, as the channel was assumed to be constant across multiple OFDM blocks. The effect of spatial correlation on the performance of SF codes was studied in [10]. The derivations in [10] were based on the assumption of a Kronecker model, in which the spatial correlations at the transmitter and the receiver are independent from each other. However, recent measurement campaigns have presented important implications and deficiencies of the Kronecker channel model [11], as it was shown that the Kronecker structure does not describe the multipath propagation channel correctly. Up to this point, we have not discussed the third dimension: time. If the channel varies randomly from one OFDM block to another, one can further exploit the diversity in the time domain by using STF coding, jointly encoding across more than one OFDM block. In [12] and [13], the performance criteria for STF-coded MIMO-OFDM systems with independent spatial fading was derived. The authors determined the maximum achievable diversity for this case, and they provided two STF code-design methods that are guaranteed to achieve 
full diversity order of the system. However, the spatial-fading correlation effect between different antenna elements was not taken into consideration.

In this paper, with a general spatial and temporal fading correlation structure, the performance of the STF-coded MIMOOFDM system is analyzed. Our analysis handles an arbitrary joint transmit-receive spatial correlation model which covers both the non-Kronecker (nonseparable) and Kronecker (separable) structures. We determine an upper bound on the diversity gain of STF-coded MIMO-OFDM systems with a general temporal and spatial correlation structure. Furthermore, we show that the STF code via mapping, designed to achieve full diversity in independent spatial fading scenarios, can be used to achieve full diversity for arbitrary correlation fading channels. Hence, the upper bound on the diversity gain is achieved. The general framework of our analysis includes SF-coded MIMOOFDM systems as a special case, hence, an upper bound on the maximum achievable diversity gain is determined for this system. For this scenario, we show that any SF code designed to achieve full diversity gain in the independent spatial fading case can achieve full diversity gain in an arbitrary spatial correlation scenario [14].

The rest of the paper is organized as follows. In Section II, the system model used in the paper is described. In Section III, we drive the performance criteria of the system in order to illustrate the parameters that determine the diversity gain of the system. In Section IV, we derive an upper bound for the maximum achievable diversity for a channel with a general temporal and spatial correlation model, and we present an STF code design that can achieve this upper bound. Furthermore, we present some special channel-correlation scenarios to give more insights on the theoretical results. Simulation results are shown in Section V, and finally, Section VI concludes the paper.

The following notations are used in the paper: $\mathbf{I}_{N}$ denotes the $N \times N$ identity matrix; $\mathbf{1}_{N \times M}$ indicates an all-ones $N \times$ $M$ matrix; $\mathbf{1}_{N}$ is an all-ones square matrix; the superscripts $T, \mathcal{H}$, and $\otimes$ represent the transpose, conjugate transpose, and element-wise conjugation, respectively; and $\otimes$ represents the Kronecker product. Finally, vec $(\mathbf{C})$ transforms a matrix $\mathbf{C}=$ $\left[\mathbf{c}_{1} \ldots \mathbf{c}_{M}\right]$ into a column vector $\operatorname{vec}(\mathbf{C})=\left[\mathbf{c}_{1}^{T} \ldots \mathbf{c}_{M}^{T}\right]^{T}$, where $\mathbf{c}_{i}$ is the $i$ th column.

\section{System ModeL}

In this paper, we consider a MIMO-OFDM system with $M_{t}$ transmit antennas, $M_{r}$ receive antennas, and $N$ subcarriers. Each pair of transmit and receive antennas has $L$ independent delay paths with the same delay profile. The channel is assumed to be static within each OFDM block period, but it may vary from one OFDM block to another. The channel impulse response between transmit antenna $i$ and receive antenna $j$ at the $k$ th OFDM block can be modeled as

$$
h_{i j}^{k}(\tau)=\sum_{l=0}^{L-1} \alpha_{i j}^{k}(l) \delta\left(\tau-\tau_{l}\right)
$$

where $\tau_{l}$ and $\alpha_{i j}^{k}(l)$ are the delay and complex amplitude of the $l$ th path between transmit antenna $i$ and receive antenna $j$, respectively. The $\alpha_{i j}^{k}(l)$ 's are modeled as zero-mean, complex
Gaussian random variables with variances $E\left|\alpha_{i j}^{k}(l)\right|^{2}=\sigma_{l}^{2}$ where $E$ stands for the expectation. The powers of the $L$ paths are normalized such that $\sum_{l=0}^{L-1} \sigma_{l}^{2}=1$. From (1), the frequency response of the channel is given by

$$
h_{i j}^{k}(f)=\sum_{l=0}^{L-1} \alpha_{i j}^{k}(l) e^{-\mathrm{j} 2 \pi f \tau_{l}}
$$

where $\mathbf{j}=\sqrt{-1}$.

The spatial and temporal fading correlations of the channel are modeled as follows. The MIMO channel is assumed to have arbitrary spatial correlation at both the transmitter and receiver sides, which will be reflected in their spatial correlation matrix later. The channel gains are assumed to be jointly Gaussian. It is also assumed that path gains for different delays are independent, i.e., different clusters of scatterers are assumed to be independent. With the assumption that the second-order statistics of the time correlation is the same for all transmit and receive antenna pairs and all paths (i.e., the correlation values do not depend on $i, j$, and $l$ ), we can define the time correlation at lag $m$ as

$$
r_{T}(m)=\frac{1}{\sigma_{l}^{2}} E\left\{\alpha_{i j}^{k}(l) \alpha_{i j}^{k-m^{*}}(l)\right\} .
$$

We assume that the spatial and time fading are independent from each other, i.e.,

$$
E\left\{\alpha_{i j}^{k}(l) \alpha_{p q}^{m^{*}}(l)\right\}=r_{T}(k-m) E\left\{\alpha_{i j}^{k}(l) \alpha_{p q}^{k^{*}}(l)\right\} .
$$

(Note that this assumption is valid for a widely used model for temporal correlation in radio communication channels [16], [17], as shown in Appendix A.)

At the transmitter, we consider STF coding across $M_{t}$ transmit antennas, $N$ OFDM subcarriers, and $K$ consecutive OFDM blocks. Each STF codeword can be expressed as an $M_{t} \times K N$ matrix

$$
\mathbf{C}=\left[\begin{array}{llll}
\mathbf{C}_{1} & \mathbf{C}_{2} & \cdots & \mathbf{C}_{K}
\end{array}\right]
$$

where the channel symbol at the $k$ th OFDM block $\mathbf{C}_{k}$ is given by an $M_{t} \times N$ matrix

$$
\mathbf{C}_{k}=\left[\begin{array}{llll}
\mathbf{c}_{k}(0) & \mathbf{c}_{k}(1) & \ldots & \mathbf{c}_{k}(N-1)
\end{array}\right]
$$

in which $\mathbf{c}_{k}(n)=\left[c_{k}^{1}(n) c_{k}^{2}(n) \ldots c_{k}^{M_{t}}(n)\right]^{T}$ is an $M_{t} \times 1$ column vector, representing the channel symbol vector transmitted on the $n$th subcarrier during the $k$ th OFDM block period, and $c_{k}^{i}(n)$ is the channel symbol transmitted over the $n$th subcarrier by transmit antenna $i$ in the $k$ th OFDM block. The STF code is assumed to satisfy the energy constraint $E\|C\|_{F}^{2}=K N M_{t}$, where $\|C\|_{F}$ is the Frobenius norm of C. During the $k$ th OFDM block period, the transmitter applies an $N$-point inverse fast Fourier transform (IFFT) to each row of the matrix $\mathbf{C}_{k}$, appends a cyclic prefix (CP), and transmits the OFDM symbol corresponding to the $i$ th $\left(i=1,2, \ldots, M_{t}\right)$ row of $\mathbf{C}_{k}$ by transmit 
antenna $i$. The $\mathrm{CP}$ is taken to be longer than the channel delay spread in order to guarantee transforming the channel to be flat-fading over each subcarrier.

At the receiver, after matched filtering, removing the $\mathrm{CP}$, and applying FFT, the received signal at the $n$th subcarrier at receive antenna $j$ in the $k$ th OFDM block is given by

$$
y_{j}^{k}(n)=\sqrt{\frac{\rho}{M_{t}}} \mathbf{h}_{j}^{k^{T}}(n) \mathbf{c}_{k}(n)+z_{j}^{k}(n)
$$

where

$$
\mathbf{h}_{j}^{k}(n)=\left[\begin{array}{llll}
H_{1 j}^{k}(n) & H_{2 j}^{k}(n) & \ldots & H_{M_{t} j}^{k}(n)
\end{array}\right]^{T}
$$

in which

$$
H_{i j}^{k}(n)=\sum_{l=0}^{L-1} \alpha_{i j}^{k}(l) e^{-\mathrm{j} 2 \pi n \Delta f \tau_{l}}
$$

is the channel frequency response at the $n$th subcarrier between transmit antenna $i$ and receive antenna $j, \Delta f=1 / T_{o}$ is the subcarrier separation in the frequency domain, and $T_{o}$ is the OFDM symbol period. In (7), $z_{j}^{k}(n)$ denotes the additive white complex Gaussian noise with zero mean and unit variance at the $n$th subcarrier at receive antenna $j$ in the $k$ th OFDM block. The factor $\sqrt{\rho / M_{t}}$ in (7) ensures that $\rho$ is the average signal-to-noise ratio (SNR) at each receive antenna.

\section{SyStem PERformancE ANALYSIS}

In this section, we analyze the performance of the STF-coded MIMO-OFDM systems with arbitrary channel correlation conditions. We derive the average pairwise error probability (PEP), which will give insights on the factors that determine the diversity order of the system performance.

The received signal during the $k$ th OFDM block in (7) can be represented in vector form as

$$
\mathbf{Y}^{k}=\sqrt{\frac{\rho}{M_{t}}} \mathbf{H}^{k} \operatorname{vec}\left(\mathbf{C}_{k}\right)+\mathbf{Z}^{k}
$$

where

$$
\mathbf{H}^{k}=\left[\begin{array}{llll}
\mathbf{H}_{1}^{k^{T}} & \mathbf{H}_{2}^{k^{T}} & \ldots & \mathbf{H}_{M_{r}}^{k^{T}}
\end{array}\right]^{T}
$$

in which $\mathbf{H}_{j}^{k}$ represents the channel frequency response to receive antenna $j$ at the $k$ th OFDM block period, and is formatted as an $N \times N M_{t}$ block diagonal matrix as follows:

$$
\mathbf{H}_{j}^{k}=\operatorname{diag}\left(\mathbf{h}_{j}^{k^{T}}(0), \mathbf{h}_{j}^{k^{T}}(1), \ldots, \mathbf{h}_{j}^{k^{T}}(N-1)\right)
$$

In (10), the vector $\mathbf{Y}^{k}$ can be written as

$$
\begin{aligned}
\mathbf{Y}^{k}=\left[y_{1}^{k}(0) \ldots y_{1}^{k}(N-1) y_{2}^{k}(0)\right. \\
\left.\quad \ldots y_{2}^{k}(N-1) \ldots y_{M_{r}}^{k}(0) \ldots y_{M_{r}}^{k}(N-1)\right]^{T}
\end{aligned}
$$

We further write the whole received signal in a compact matrix form as

$$
\mathbf{Y}=\sqrt{\frac{\rho}{M_{t}}} \mathbf{H v e c}(\mathbf{C})+\mathbf{Z}
$$

where

$$
\mathbf{H}=\operatorname{diag}\left(\mathbf{H}^{1}, \quad \mathbf{H}^{2}, \quad \cdots \quad \mathbf{H}^{K}\right)
$$

and the received $K N M_{r} \times 1$ signal vector $\mathbf{Y}$ is given by

$$
\mathbf{Y}=\left[\begin{array}{llll}
\mathbf{Y}^{1^{T}} & \mathbf{Y}^{2^{T}} & \cdots & \mathbf{Y}^{M_{r}^{T}}
\end{array}\right]^{T}
$$

The receiver is assumed to know the channel state information exactly, and thus it can apply a maximum-likelihood decoder

$$
\hat{\mathbf{C}}=\underset{\mathbf{C}}{\operatorname{argmin}}\left\|\mathbf{Y}-\sqrt{\frac{\rho}{M_{t}}} \mathbf{H} \operatorname{vec}(\mathbf{C})\right\|^{2} .
$$

Accordingly, the PEP between two codewords $\mathbf{C}$ and $\tilde{\mathbf{C}}$ for a given channel realization can be upper bounded by [18]

$$
\operatorname{Pr}(\mathbf{C} \rightarrow \tilde{\mathbf{C}} \mid \mathbf{H}) \leq \frac{1}{2} \exp \left(-\frac{\rho}{4 M_{t}}\|\boldsymbol{\Phi}\|^{2}\right)
$$

where $\boldsymbol{\Phi}$ is a $K N M_{r} \times 1$ vector given by

$$
\boldsymbol{\Phi}=\mathbf{H}[\operatorname{vec}(\mathbf{C})-\operatorname{vec}(\tilde{\mathbf{C}})]
$$

Since the channel coefficients are jointly Gaussian, the vector $\Phi$, for a fixed code realization, has a Gaussian distribution with zero mean and covariance matrix

$$
\mathbf{R}_{\boldsymbol{\Phi}}=E\left\{\boldsymbol{\Phi} \boldsymbol{\Phi}^{\mathcal{H}}\right\}
$$

of size $K N M_{r} \times K N M_{r}$. Since $N$ is usually greater than $L M_{t}$, the matrix $\mathbf{R}_{\boldsymbol{\Phi}}$ can be shown to be rank-deficient. Averaging the PEP (18) over all channel realizations, we get [19]

$$
\operatorname{Pr}(\mathbf{C} \rightarrow \tilde{\mathbf{C}}) \leq\left(\frac{\rho}{4 M_{t}}\right)^{-r\left(\mathbf{R}_{\boldsymbol{\Phi}}\right)}\left(\prod_{i=0}^{-r\left(\mathbf{R}_{\boldsymbol{\Phi}}\right)-1} \lambda_{i}\left(\mathbf{R}_{\boldsymbol{\Phi}}\right)^{-1}\right)
$$


where $r\left(\mathbf{R}_{\boldsymbol{\Phi}}\right)$ and $\lambda_{i}\left(\mathbf{R}_{\boldsymbol{\Phi}}\right)$ are the rank and the $i$ th eigenvalue of the covariance matrix $\mathbf{R}_{\Phi}$, respectively.

\section{MAXIMUM ACHIEVABLE DiVERSITY}

We analyze in this section the factors that affect the diversity order of an STF-coded MIMO-OFDM system. First, we derive an upper bound for the maximum achievable diversity for such a system. Second, we show that this bound can be achieved by a repetition STF code design. Finally, we investigate the general analytical results that we derived for some special correlation scenarios of practical interest.

\section{A. An Upper Bound for the Maximum Achievable Diversity Order}

In this section, we determine an upper bound for the maximum achievable diversity order of the STF-coded MIMO-OFDM system with an arbitrary spatial and temporal correlation structure, as specified in Section II. From the PEP in (21), we can see that the diversity order is determined by the rank of the covariance matrix $\mathbf{R}_{\Phi}$ in (20). Next, we further determine the covariance matrix $\mathbf{R}_{\boldsymbol{\Phi}}$.

From (19), the covariance matrix $\mathbf{R}_{\boldsymbol{\Phi}}$ can be written as

$$
\begin{aligned}
\mathbf{R}_{\boldsymbol{\Phi}} & =E\left[\mathbf{H v e c}(\mathbf{C}-\tilde{\mathbf{C}}) \operatorname{vec}^{\mathcal{H}}(\mathbf{C}-\tilde{\mathbf{C}}) \mathbf{H}^{\mathcal{H}}\right] \\
& =E\left(\mathbf{H} \boldsymbol{\Delta} \boldsymbol{\Delta}^{\mathcal{H}} \mathbf{H}^{\mathcal{H}}\right)
\end{aligned}
$$

where $\boldsymbol{\Delta}=\operatorname{vec}(\mathbf{C}-\tilde{\mathbf{C}})$. Using (5) and (15), we can write (22) as

$$
\mathbf{R}_{\boldsymbol{\Phi}}=E\left\{\left[\begin{array}{c}
\mathbf{H}^{1} \Delta^{1} \\
\vdots \\
\mathbf{H}^{K} \Delta^{K}
\end{array}\right]\left[\begin{array}{lll}
\Delta^{1^{\mathcal{H}}} \mathbf{H}^{1^{\mathcal{H}}} & \ldots & \Delta^{K^{\mathcal{H}}} \mathbf{H}^{K^{\mathcal{H}}}
\end{array}\right]\right\}
$$

where $\boldsymbol{\Delta}^{j}=\operatorname{vec}\left(\mathbf{C}_{j}-\tilde{\mathbf{C}}_{j}\right), 1 \leq j \leq K$.

Define $\mathbf{R}_{\boldsymbol{\Phi}_{n m}} \triangleq E\left[\mathbf{H}^{n} \boldsymbol{\Delta}^{n} \boldsymbol{\Delta}^{m^{\mathcal{H}}} \mathbf{H}^{m^{\mathcal{H}}}\right]$, then we can write $\mathbf{R}_{\boldsymbol{\Phi}_{n m}}$ as

$$
\mathbf{R}_{\boldsymbol{\Phi}_{n m}}=E\left\{\left[\begin{array}{c}
\mathbf{H}_{1}^{n} \Delta^{n} \\
\vdots \\
\mathbf{H}_{M_{r}}^{n} \Delta^{n}
\end{array}\right]\left[\begin{array}{llll}
\Delta^{m^{\mathcal{H}}} \mathbf{H}_{1}^{m^{\mathcal{H}}} & \ldots & \Delta^{m^{\mathcal{H}}} \mathbf{H}_{M_{r}}^{m^{\mathcal{H}}}
\end{array}\right]\right\} .
$$

Denoting $E\left[\mathbf{H}_{j}^{n} \Delta^{n} \boldsymbol{\Delta}^{m^{\mathcal{H}}} \mathbf{H}_{p}^{m^{\mathcal{H}}} \mathbf{H}_{p}^{m^{\mathcal{H}}}\right]$ as $\mathbf{A}_{j p}^{n m}$, we can compute the value of this matrix using (12) as follows:

$$
\begin{array}{r}
\mathbf{A}_{j p}^{n m}=E\left\{[ \begin{array} { c } 
{ \Delta ^ { n ^ { T } } ( 0 ) \mathbf { h } _ { \mathbf { j } } ^ { \mathbf { n } } ( 0 ) } \\
{ \vdots } \\
{ \Delta ^ { n ^ { T } } ( N - 1 ) \mathbf { h } _ { \mathbf { j } } ^ { \mathbf { n } } ( N - 1 ) }
\end{array} ] \left[\mathbf{h}_{p}^{m^{\mathcal{H}}}(0) \Delta^{m^{*}}(0) \ldots\right.\right. \\
\left.\left.\mathbf{h}_{p}^{m^{\mathcal{H}}}(N-1) \Delta^{m^{*}}(N-1)\right]\right\}
\end{array}
$$

where $\Delta^{i}(n)=\left(\mathbf{c}_{i}(n)-\tilde{\mathbf{c}}_{i}(n)\right)$. Using (8) and (9), the vector $\mathbf{h}_{j}^{i}$ can be written as $\mathbf{h}_{j}^{j}(n)=\sum_{l=0}^{L-1} e^{-j 2 \pi n \Delta f \tau_{l}} \alpha_{j}^{i}(l)$, in which $\alpha_{j}^{i}(l)=\left[\begin{array}{llll}\alpha_{1 j}^{i}(l) & \ldots & \alpha_{M_{t} j}^{i}(l)\end{array}\right]^{T}$. With the assumption that different delay paths are independent, we have

$$
E\left[\mathbf{h}_{j}^{n}(n) \mathbf{h}_{p}^{m^{\mathcal{H}}}(k)\right]=\sum_{l=0}^{L-1} e^{-j 2 \pi(n-k) \Delta f \tau_{l}} E\left[\alpha_{j}^{n}(l) \alpha_{p}^{m^{\mathcal{H}}}(l)\right] .
$$

From (25) and (26), we have $\mathbf{A}_{j p}^{n m}$ as follows:

$$
\begin{gathered}
\mathbf{A}_{j p}^{n m}=\sum_{l=0}^{L-1} E\left\{\left[\begin{array}{c}
\Delta^{n^{T}}(0) \alpha_{j}^{n}(l) \\
\vdots \\
e^{-j 2 \pi(N-1) \Delta f \tau_{l} \Delta^{n^{T}}(N-1) \alpha_{j}^{n}(l)}
\end{array}\right]\right. \\
\cdot\left[\alpha_{p}^{m^{\mathcal{H}}}(l) \Delta^{m^{*}}(0) \ldots \alpha_{p}^{m^{\mathcal{H}}}(l) \Delta^{m^{*}}(N-1)\right. \\
\left.\left.\cdot e^{j 2 \pi(N-1) \Delta f \tau_{l}}\right]\right\} .
\end{gathered}
$$

Using the fact that $\Delta^{i}(n)$ is the $n$th column of the matrix $\left(\mathbf{C}_{i}-\right.$ $\tilde{\mathbf{C}}_{i}$ ), we can further simplify (27) as follows:

$$
\begin{aligned}
\mathbf{A}_{j p}^{n m}=\sum_{l=0}^{L-1} \mathbf{D}^{\tau_{l}} & \left(\mathbf{C}_{n}-\tilde{\mathbf{C}}_{n}\right)^{T} E \\
& \times\left[\alpha_{j}^{n}(l) \alpha_{p}^{m^{\mathcal{H}}}(l)\right]\left(\mathbf{C}_{m}-\tilde{\mathbf{C}}_{m}\right)^{*} \mathbf{D}^{* \tau_{l}}
\end{aligned}
$$

where

$$
\mathbf{D}=\operatorname{diag}\left(1, e^{-j 2 \pi \Delta f}, \ldots, e^{-j 2 \pi(N-1) \Delta f}\right) .
$$

Using (4), and combining (24) and (28), we get

$$
\begin{array}{r}
\mathbf{R}_{\boldsymbol{\Phi}_{n m}}=r_{T}(n-m) \sum_{l=0}^{L-1}\left[\mathbf{I}_{M_{r}}-\otimes \mathbf{D}^{\tau_{l}}\left(\mathbf{C}_{n}-\tilde{\mathbf{C}}_{n}\right)^{T}\right] \\
\times \mathbf{R}_{\alpha}(l)\left[\mathbf{I}_{M_{r}} \otimes\left(\mathbf{C}_{m}-\tilde{\mathbf{C}}_{m}\right)^{*} \mathbf{D}^{* \tau_{l}}\right]
\end{array}
$$

where the matrix $\mathbf{R}_{\alpha}(l)$ is the spatial covariance matrix of the $l$ th path (lth cluster) of the channel impulse response, and is specified as

$$
\mathbf{R}_{\alpha}(l)=E\left[\alpha(l) \alpha^{\mathcal{H}}(l)\right]
$$

in which the vector $\alpha(l)=\left[\alpha_{11}(l) \ldots \alpha_{M_{t} 1}(l) \ldots \alpha_{1 M_{r}}(l)\right.$ $\left.\ldots \alpha_{M_{t} M_{r}}(l)\right]^{T}$ has size $M_{r} M_{t} \times 1$. Define the $N M_{r} \times L M_{t} M_{r}$ matrix $\mathbf{F}_{i}$ as

$\mathbf{F}_{i}=\left[\mathbf{I}_{M_{r}} \otimes \mathbf{D}^{\tau_{0}}\left(\mathbf{C}_{i}-\tilde{\mathbf{C}}_{i}\right)^{T}, \cdots, \mathbf{I}_{M_{r}} \otimes \mathbf{D}^{\tau_{L-1}}\left(\mathbf{C}_{i}-\tilde{\mathbf{C}}_{i}\right)^{T}\right]$ 
Hence, we can write (30) in a compact form as follows:

$$
\mathbf{R}_{\boldsymbol{\Phi}_{n m}}=r_{T}(n-m) \mathbf{F}_{n} \mathbf{R}_{\alpha} \mathbf{F}_{m}^{\mathcal{H}}
$$

where the matrix $\mathbf{R}_{\alpha}$ is an $L M_{t} M_{r} \times L M_{t} M_{r}$ block diagonal matrix, and is given by

$$
\mathbf{R}_{\alpha}=\operatorname{diag}\left(\mathbf{R}_{\alpha}(0) \quad \ldots \quad \mathbf{R}_{\alpha}(L-1)\right) .
$$

Using (33), we can write the whole matrix $\mathbf{R}_{\Phi}$ as follows:

$$
\mathbf{R}_{\boldsymbol{\Phi}}=\left[\begin{array}{lrr}
r_{T}(0) \mathbf{F}_{1} \mathbf{R}_{\alpha} \mathbf{F}_{1}^{\mathcal{H}} & \cdots & r_{T}(1-K) \mathbf{F}_{1} \mathbf{R}_{\alpha} \mathbf{F}_{K}^{\mathcal{H}} \\
r_{T}(1) \mathbf{F}_{2} \mathbf{R}_{\alpha} \mathbf{F}_{1}^{\mathcal{H}} & \cdots & r_{T}(2-K) \mathbf{F}_{2} \mathbf{R}_{\alpha} \mathbf{F}_{K}^{\mathcal{H}} \\
\vdots & \ddots & \vdots \\
r_{T}(K-1) \mathbf{F}_{K} \mathbf{R}_{\alpha} \mathbf{F}_{1}^{\mathcal{H}} & \cdots & r_{T}(0) \mathbf{F}_{K} \mathbf{R}_{\alpha} \mathbf{F}_{K}^{\mathcal{H}}
\end{array}\right] .
$$

The matrix $\mathbf{F}_{n} \mathbf{R}_{\alpha} \mathbf{F}_{m}^{\mathcal{H}}$ is of size $N M_{r} \times N M_{r}$, thus we can write (35) as

$$
\begin{array}{r}
\mathbf{R}_{\boldsymbol{\Phi}}=\left[\begin{array}{lrrr}
r_{T}(0) \mathbf{1}_{N M_{r}} & \cdots & r_{T}(1-K) \mathbf{1}_{N M_{r}} \\
r_{T}(1) \mathbf{1}_{N M_{r}} & \cdots & r_{T}(2-K) \mathbf{1}_{N M_{r}} \\
\vdots & \ddots & & \vdots \\
r_{T}(K-1) \mathbf{1}_{N M_{r}} & \cdots & \multicolumn{2}{r}{r_{T}(0) \mathbf{1}_{N M_{r}}}
\end{array}\right] \\
\circ\left[\begin{array}{lllr}
\mathbf{F}_{1} \mathbf{R}_{\alpha} \mathbf{F}_{1}^{\mathcal{H}} & \cdots & \mathbf{F}_{1} \mathbf{R}_{\alpha} \mathbf{F}_{K}^{\mathcal{H}} \\
\mathbf{F}_{2} \mathbf{R}_{\alpha} \mathbf{F}_{1}^{\mathcal{H}} & \cdots & \mathbf{F}_{2} \mathbf{R}_{\alpha} \mathbf{F}_{K}^{\mathcal{H}} \\
\vdots & & \ddots & \vdots \\
\mathbf{F}_{K} \mathbf{R}_{\alpha} \mathbf{F}_{1}^{\mathcal{H}} & \cdots & \mathbf{F}_{K} \mathbf{R}_{\alpha} \mathbf{F}_{K}^{\mathcal{H}}
\end{array}\right]
\end{array}
$$

where $\circ$ denotes the Hadamard product. ${ }^{1}$ From the above analysis, the covariance matrix $\mathbf{R}_{\Phi}$ can be further decomposed in the following theorem.

Theorem 1: The covariance matrix $\mathbf{R}_{\boldsymbol{\Phi}}$ in (20) can be decomposed as

$$
\begin{array}{r}
\mathbf{R}_{\boldsymbol{\Phi}}=\left(R_{T} \otimes \mathbf{1}_{N M_{r}}\right) \circ\left[\operatorname{diag}\left(\mathbf{F}_{1}, \mathbf{F}_{2}, \ldots, \mathbf{F}_{K}\right)\left(\mathbf{1}_{k} \otimes \mathbf{R}_{\alpha}\right)\right. \\
\left.\times \operatorname{diag}\left(\mathbf{F}_{1}, \mathbf{F}_{2}, \ldots, \mathbf{F}_{K}\right)^{\mathcal{H}}\right]
\end{array}
$$

where the matrix $\mathbf{R}_{T}$ is the temporal correlation matrix of size $K \times K$, whose entry in the $p$ th row and the $q$ th column is given by $r_{T}(p-q)$ for $1 \leq p, q \leq K$.

Theorem 1 determines the factors that affect the diversity order of an STF-coded MIMO-OFDM system with an arbitrary temporal and spatial correlation structure. The theorem identifies the relation between the matrix $\mathbf{R}_{\boldsymbol{\Phi}}$, whose rank determines the diversity order of the system, and the temporal correlation matrix $\mathbf{R}_{\mathbf{T}}$, the spatial correlation matrix $\mathbf{R}_{\alpha}$, and the STF code used $\left(F_{i}, 1 \leq i \leq K\right)$.

Next, we derive an upper bound on the maximum achievable diversity order of the STF-coded MIMO-OFDM system with

\footnotetext{
${ }^{1}$ Suppose that $A=\left[a_{i, j}\right]$ and $B=\left[b_{i, j}\right]$ are two matrices of size $m \times n$. The Hadamard product of $A$ and $B$ is defined as the element-wise matrix product $A \circ B=\left[a_{i, j} b_{i, j_{1 \leq i \leq m, 1 \leq j \leq n}}\right]$.
}

the arbitrary spatial and temporal correlation structure. The matrix $\mathbf{R}_{\Phi}$ in (37) comes from the Hadamard product of two matrices, and thus has rank

$$
\begin{array}{r}
r\left(\mathbf{R}_{\boldsymbol{\Phi}}\right) \leq r\left(\mathbf{R}_{T}\right) r\left\{\operatorname{diag}\left(\mathbf{F}_{1}, \mathbf{F}_{2}, \ldots, \mathbf{F}_{K}\right)\left(\mathbf{1}_{k} \otimes \mathbf{R}_{\alpha}\right)\right. \\
\left.\times \operatorname{diag}\left(\mathbf{F}_{1}, \mathbf{F}_{2}, \cdots, \mathbf{F}_{K}\right)^{\mathcal{H}}\right\} .
\end{array}
$$

If the STF code is designed to achieve full diversity in the independent spatial fading channel case, then the $K N M_{r} \times K L M_{t} M_{r}$ matrix $\mathbf{G}=\operatorname{diag}\left(\mathbf{F}_{1}, \mathbf{F}_{2}, \ldots, \mathbf{F}_{K}\right)$, in the right-hand side of (38), can be shown to be of rank $K L M_{t} M_{r}$ [12], [13]. Denote $\mathbf{G}\left(\mathbf{1}_{k} \otimes \mathbf{R}_{\alpha}\right) \mathbf{G}^{\mathcal{H}}$ by $\mathbf{J}$, which can be rewritten after row and column reordering in the form

$$
\begin{aligned}
\tilde{\mathbf{J}} & =\left[\begin{array}{l}
\mathbf{G}_{1} \\
\mathbf{G}_{2}
\end{array}\right]\left(\mathbf{1}_{k} \otimes \mathbf{R}_{\alpha}\right)\left[\begin{array}{ll}
\mathbf{G}_{1}^{\mathcal{H}} & \mathbf{G}_{2}^{\mathcal{H}}
\end{array}\right] \\
& =\left[\begin{array}{ll}
\mathbf{G}_{1}\left(\mathbf{1}_{k} \otimes \mathbf{R}_{\alpha}\right) \mathbf{G}_{1}^{\mathcal{H}} & \mathbf{G}_{1}\left(\mathbf{1}_{k} \otimes \mathbf{R}_{\alpha}\right) \mathbf{G}_{2}^{\mathcal{H}} \\
\mathbf{G}_{2}\left(\mathbf{1}_{k} \otimes \mathbf{R}_{\alpha}\right) \mathbf{G}_{1}^{\mathcal{H}} & \mathbf{G}_{2}\left(\mathbf{1}_{k} \otimes \mathbf{R}_{\alpha}\right) \mathbf{G}_{2}^{\mathcal{H}}
\end{array}\right]
\end{aligned}
$$

where $\mathbf{G}_{1}$ is of size $K L M_{t} M_{r} \times K L M_{t} M_{r}$, and is full rank. The matrix $\mathbf{G}_{2}$ takes the rest of the matrix. Since the ordered singular values of a matrix are not smaller than the corresponding singular values of any square submatrix obtained by deleting equal number of rows and columns of the original matrix [20], we get

$$
\lambda_{i}(\mathbf{J}) \geq \lambda_{i}\left(\mathbf{G}_{1}\left(\mathbf{1}_{k} \otimes \mathbf{R}_{\alpha}\right) \mathbf{G}_{1}^{\mathcal{H}}\right)
$$

where $\lambda_{i}(\cdot)$ denotes the $i$ th eigenvalue, and they are ordered in decreasing order. The eigenvalues of $\mathbf{G}_{1}\left(\mathbf{1}_{k} \otimes \mathbf{R}_{\alpha}\right) \mathbf{G}_{l}^{\mathcal{H}}$ are given by

$$
\left.\lambda_{i}\left(\mathbf{G}_{1}\left(\mathbf{1}_{k} \otimes \mathbf{R}_{\alpha}\right) \mathbf{G}_{1}^{\mathcal{H}}\right)=\theta_{i} \lambda_{i}\left(\mathbf{1}_{k} \otimes \mathbf{R}_{\alpha}\right)\right)
$$

where $\theta_{i}$ is a nonnegative real number, such that $\lambda_{1}\left(\mathbf{G}_{1} \mathbf{G}_{1}^{\mathcal{H}}\right) \leq$ $\theta_{i} \leq \lambda_{K L M_{t} M_{r}}\left(\mathbf{G}_{1} \mathbf{G}_{1}^{\mathcal{H}}\right)$, which follows by Ostrowski's theorem [21]. Thus, the rank of the matrix $\mathbf{J}$ is given by

$$
r(\mathbf{J})=r\left(\mathbf{1}_{\mathbf{k}} \otimes \mathbf{R}_{\alpha}\right)=r\left(\mathbf{R}_{\alpha}\right) .
$$

Substituting (42) in (38), we get

$$
r\left(\mathbf{R}_{\Phi}\right) \leq r\left(\mathbf{R}_{T}\right) \cdot r\left(\mathbf{R}_{\alpha}\right) .
$$

We summarize this result in the following theorem.

Theorem 2: The maximum achievable diversity order of the STF-coded MIMO-OFDM system with arbitrary spatial and temporal correlation channels, as described in (3), (4), and (34), is upper bounded by

$$
r\left(\mathbf{R}_{\Phi}\right) \leq r\left(\mathbf{R}_{T}\right) \cdot r\left(\mathbf{R}_{\alpha}\right) .
$$




\section{B. STF Code Design to Achieve the Diversity Upper Bound}

In Theorem 2, we have proved an upper bound for the diversity order of an STF-coded MIMO-OFDM system, but we did not discuss whether this bound is achieved or not. In this section, we show that the repetition STF code design, proposed in [12] and [13], can indeed achieve full diversity order in arbitrary correlated channel scenarios, and this proves that the inequality in Theorem 2 is actually with equality.

In [12] and [13], a systematic approach to the design of fulldiversity STF codes was proposed. An STF code is constructed by repeating a full-diversity SF code $K$ times over $K$ OFDM blocks, as follows:

$$
\mathrm{C}=\mathbf{1}_{1 \times K} \otimes \mathbf{C}_{\mathrm{SF}}
$$

where $\mathbf{C}_{\mathrm{SF}}$ is a full-diversity $\mathrm{SF}$ code. In this case, the covariance matrix $\mathbf{R}_{\boldsymbol{\Phi}}$ (37) is given by

$$
\mathbf{R}_{\boldsymbol{\Phi}}=\left[\begin{array}{ccc}
r_{T}(0) \mathbf{F} \mathbf{R}_{\alpha} \mathbf{F}^{\mathcal{H}} & \cdots & r_{T}(1-K) \mathbf{F} \mathbf{R}_{\alpha} \mathbf{F}^{\mathcal{H}} \\
r_{T}(1) \mathbf{F} \mathbf{R}_{\alpha} \mathbf{F}^{\mathcal{H}} & \cdots & r_{T}(2-K) \mathbf{F} \mathbf{R}_{\alpha} \mathbf{F}^{\mathcal{H}} \\
\vdots & \ddots & \vdots \\
r_{T}(K-1) \mathbf{F} \mathbf{R}_{\alpha} \mathbf{F}^{\mathcal{H}} & \cdots & r_{T}(0) \mathbf{F} \mathbf{R}_{\alpha} \mathbf{F}^{\mathcal{H}}
\end{array}\right]
$$

where $\mathbf{F}=\left[\mathbf{I}_{M_{r}} \otimes \mathbf{D}^{\tau_{0}}\left(\mathbf{C}_{\mathrm{SF}}-\tilde{\mathbf{C}}_{\mathrm{SF}}\right)^{T} \ldots \mathbf{I}_{M_{r}} \otimes \mathbf{D}^{\tau_{L-1}}\left(\mathbf{C}_{\mathrm{SF}}-\right.\right.$ $\left.\tilde{\mathbf{C}}_{\mathrm{SF}}\right)^{T}$. Equation (46) can be written as

$$
\mathbf{R}_{\boldsymbol{\Phi}}=\mathbf{R}_{T} \otimes\left(\mathbf{F} \mathbf{R}_{\alpha} \mathbf{F}^{\mathcal{H}}\right) .
$$

The rank of the matrix $\mathbf{R}_{\boldsymbol{\Phi}}$ in this case is given by

$$
r\left(\mathbf{R}_{\Phi}\right)=r\left(\mathbf{R}_{T}\right) \cdot r\left(\mathbf{F R}_{\alpha} \mathbf{F}^{\mathcal{H}}\right)
$$

where the equality here is due to the Kronecker product. Following similar arguments as in (39)-(42), we can show that $r\left(\mathbf{F} \mathbf{R}_{\alpha} \mathbf{F}^{\mathcal{H}}\right)=r\left(\mathbf{R}_{\alpha}\right)$. Hence, the diversity gain of the STFMIMO-OFDM system is given by

$$
r\left(\mathbf{R}_{\Phi}\right)=r\left(\mathbf{R}_{T}\right) \cdot r\left(\mathbf{R}_{\alpha}\right) .
$$

We conclude from this that the upper bound of the diversity order in Theorem 2 can indeed be achieved. The repetitioncoded STF code design guarantees full diversity in arbitrary spatial and temporal correlation at the price of symbol-rate reduction by a factor of $1 / K$, compared with the symbol rate of the underlying SF code used.

Discussion: Since the spatial covariance matrix $\mathbf{R}_{\alpha}$ in (34) is a diagonal matrix, we can further determine the maximum achievable diversity of the STF-coded MIMO-OFDM system as follows:

$$
r\left(\mathbf{R}_{\boldsymbol{\Phi}}\right)=r\left(\mathbf{R}_{T}\right) \sum_{l=0}^{L-1} r\left(\mathbf{R}_{\alpha}(l)\right) .
$$

The physical interpretation behind (50) is that the maximum number of independent replicas of the signal that can be achieved at the receiver, i.e., the diversity gain, depends on the temporal, spatial, and multipath-delay diversity inherent in the system. Since we have assumed in (4) that the spatial and temporal fading are independent, then the total number of independent channels will be a product of the number of independent channels we can achieve from each of them. The DOFs for the time domain are given by the rank of the temporal covariance matrix $\mathbf{R}_{T}$. For the multipath-delay environment, we have $L$ independent clusters, thus, the independent replicas at the receiver will be equal to the sum of independent replicas emanating from each cluster. The number of independent replicas from the $l$ th cluster is just the rank of $\mathbf{R}_{\alpha}(l)$, the spatial covariance matrix for that cluster, and this interprets the result in $(50)$.

\section{Maximum Achievable Diversity for Some Special Correlation Scenarios}

We determined above the maximum achievable diversity for the general correlated fading scenario. In the rest of this section, we interpret the system analytical results for some special correlation scenarios of practical interest. As we will see, the obtained results are consistent with the existing results in the literature.

Case 1: Space-Frequency Scenario: SF coding, where coding is done across multiple antennas and all subcarriers within each OFDM block, can be considered as a special case of STF coding, because only one OFDM block period $(K=1)$ is considered. The temporal covariance matrix $\mathbf{R}_{T}$ degrades in this case, and the maximum achievable diversity for the SF-coded MIMO-OFDM system is thus given by

$$
r\left(\mathbf{R}_{\boldsymbol{\Phi}}\right)=r\left(\mathbf{F R}_{\alpha} \mathbf{F}^{\mathcal{H}}\right) .
$$

In this case, any SF code that can achieve full diversity in an independent fading environment will result in the matrix $\mathbf{F}$ being full column rank. Hence, the maximum achievable diversity of the SF-coded MIMO-OFDM system is given by

$$
r\left(\mathbf{R}_{\boldsymbol{\Phi}}\right)=\sum_{l=0}^{L-1} r\left(\mathbf{R}_{\alpha}(l)\right)
$$

which agrees with [14]. This means that the diversity order of the system is equal to the number of DOFs offered by the independent scatterers. We proved in [14] that any SF code designed to achieve full diversity in the independent spatial fading channel can achieve full diversity for an arbitrary joint transmit-receive correlation channel model. The proof in [14] has some reminiscence to the analysis we did for the STF-coded case, hence, we do not repeat it here for lack of space.

Case 2: Temporal Correlation Only: If the antenna elements at both the transmitter and the receiver are well separated, one can consider no spatial correlation between fades of different transmit and receive antenna pairs (i.e., independent spatial fading). The rank of each $\mathbf{R}_{\alpha}(l)$ for this case is $M_{t} M_{r}$. Hence, the maximum achievable diversity is given by

$$
r\left(\mathbf{R}_{\Phi}\right)=r\left(\mathbf{R}_{T}\right) L M_{t} M_{r}
$$

which agrees with the results obtained in [12] and [13]. 
Case 3: Constant Channel: If the channel does not change with time, the temporal covariance matrix $\mathbf{R}_{T}$ is an all-one matrix having rank 1 , and the maximum achievable diversity will be the same as for the case of the SF-coded system (52). This scenario generally arises when the channel can be considered quasi-static over multiple OFDM block periods. As a result, there is no additional diversity gain achieved in applying STF over SF-coded systems.

Case 4: Independent Fading Channel: If there is no correlation among channel fades in both the temporal and spatial domains, the temporal correlation matrix $\mathbf{R}_{\mathbf{T}}$ has rank $K$, and the spatial correlation matrix has rank $L M_{t} M_{r}$. It is obvious, in this case, that we are able to achieve the total number of independent DOFs inherent in the physical structure of the system, which is given by

$$
r\left(\mathbf{R}_{\Phi}\right)=K L M_{t} M_{r}
$$

\section{Simulation Results}

In this section, we provide some simulation results to demonstrate our theoretical analysis. We first study the effect of spatial correlation on the performance of a system using SF codes. Then we show the effect of both spatial and temporal correlation on the performance of an STF-coded system. We present average bit-error rate (BER) curves as functions of the average SNR per bit in decibels.

\section{A. Effect of Spatial Correlation on SF Codes}

In this subsection, we show the effect of spatial correlation on the system performance. Two scenarios of an SF-coded MIMOOFDM system are considered: 1) two transmit and one receive antennas; 2) two transmit and two receive antennas. In both cases, we consider a two-ray, equal-power delay profile, with a delay of $20 \mu$ s between the two rays. The MIMO-OFDM system has $N=128$ subcarriers, and the total bandwidth is $1 \mathrm{MHz}$. We choose the full-diversity SF code from [8] to conduct our simulations. The $2 \times 2$ Alamouti structure [15] is transmitted twice (repetition two) (45) to guarantee full diversity in the case of a spatially independent fading channel. Quaternary phase-shift keying (QPSK) modulation is used to achieve a spectral efficiency of $1 \mathrm{~b} / \mathrm{s} / \mathrm{Hz}$, ignoring the $\mathrm{CP}$.

To generate the spatial correlated channel coefficients, we use the following model:

$$
\alpha_{l}=\mathbf{A}_{l} \tilde{\alpha}_{l}
$$

where $l \in\{0,1, \ldots, L-1\}, \tilde{\alpha}_{l}$ is an $M_{r} M_{t} \times 1$ vector with independent, identically distributed (i.i.d.) entries chosen from a complex Gaussian distribution with zero mean and $\sigma_{l}^{2}$ variance, and the matrix $\mathbf{A}_{l}$ contains the correlation coefficients. It is clear that the rank of $\mathbf{R}_{\alpha}(l)$ is equal to that of $\mathbf{A}_{l}$. For brevity, we will give two examples for the matrix $\mathbf{A}_{l}$ used in the simulations.

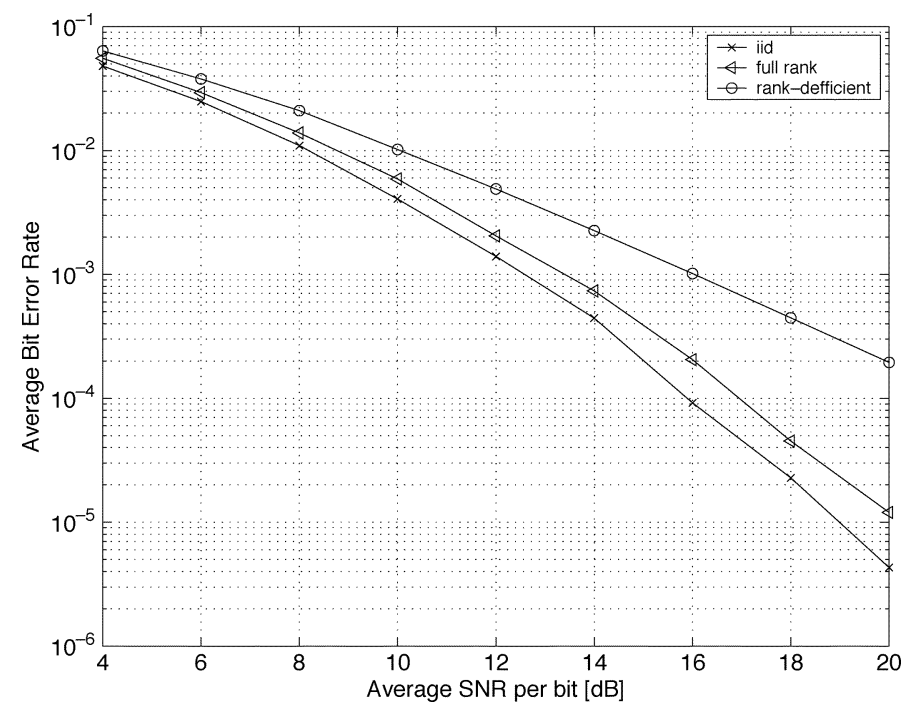

Fig. 1. BER performance of an SF-coded system with $M_{t}=2$ transmit and $M_{r}=1$ receive antennas under different spatial correlation conditions.

We simulated first an SF-coded MIMO-OFDM system with $M_{t}=2$ transmit and $M_{r}=1$ receive antennas. We compare three different correlation scenarios: 1) the independent fading case, in which $\mathbf{A}_{l}=\mathbf{I}_{2}$, thus the maximum achievable diversity is four; 2) the spatial correlation case, with $\mathbf{A}_{l}$ being full rank, thus the maximum achievable diversity is also four; and 3) the spatial correlation case, with $\mathbf{A}_{l}$, has rank $=1$, thus the maximum achievable diversity is two. An example for this case is

$$
\mathbf{A}_{0}=\left[\begin{array}{cc}
-0.07997-\mathbf{j} 0.91094 & -0.00934+\mathbf{j} 0.40459 \\
-0.65107+\mathbf{j} 0.64212 & 0.31776-\mathbf{j} 0.25062
\end{array}\right] .
$$

Fig. 1 depicts the performance of the SF code for these three cases. This figure shows that the performance curves for both the independent fading and spatial correlation with full rank cases have the same slope, hence, they achieve equal diversity gain. Also, both of these curves have a steeper slope than that of the rank-deficient case.

We also simulated an SF-coded system with $M_{t}=2$ transmit and $M_{r}=2$ receive antennas. In this setup, we compare four different correlation scenarios: 1) the independent fading case, in which $\mathbf{A}_{l}=\mathbf{I}_{4}$, thus the maximum achievable diversity is eight; 2) the spatial correlation case, with $\mathbf{A}_{l}$ being full rank, thus the maximum achievable diversity is eight; 3 ) the spatial correlation case, with $\mathbf{A}_{l}$, has rank $=2$, thus the maximum achievable diversity is four; and 4) the spatial correlation case, with $\mathbf{A}_{l}$, has rank $=1$, thus the maximum achievable diversity is two. An example of $\mathbf{A}_{0}$ for this case is shown in (57) at the bottom of the page. Fig. 2 depicts the performance of the SF code for these four cases. The figure shows that the performance curves for both the independent fading and spatial correlation

$$
\mathbf{A}_{0}=\left[\begin{array}{cccc}
-0.41-\mathbf{j} 0.25 & 0.55-\mathbf{j} 0.47 & 0.17-\mathbf{j} 0.34 & -0.27+\mathbf{j} 0.06 \\
0.43-\mathbf{j} 0.23 & 0.13+\mathbf{j} 0.72 & 0.21+\mathbf{j} 0.33 & 0.09-\mathbf{j} 0.27 \\
0.37+\mathbf{j} 0.31 & -0.62+\mathbf{j} 0.39 & -0.23+\mathbf{j} 0.31 & 0.28-\mathbf{j} 0.02 \\
-0.28-\mathbf{j} 0.39 & 0.69-\mathbf{j} 0.22 & 0.30-\mathbf{j} 0.25 & -0.27-\mathbf{j} 0.05
\end{array}\right]
$$




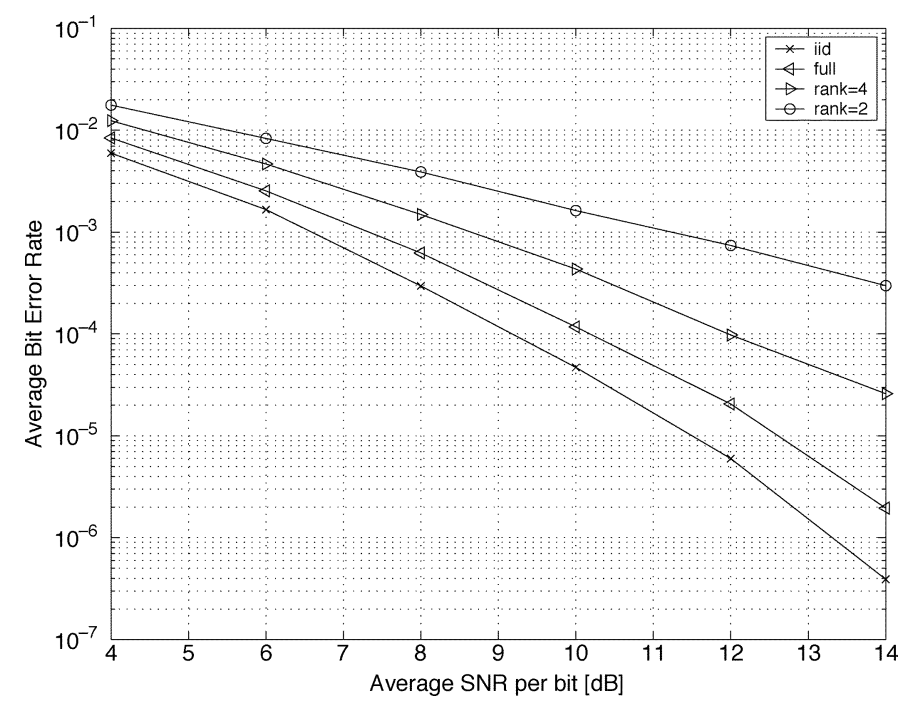

Fig. 2. BER performance of an SF-coded system with $M_{t}=2$ transmit and $M_{r}=2$ receive antennas under different spatial correlation conditions.

with full rank cases have the same slope, thus, they achieve equal diversity gain. Also, the slope of the performance curve decreases for the rank-deficient cases.

From the simulated results in Figs. 1 and 2, we observe that the SF code designed for the independent fading channel is able to achieve the maximum diversity offered in the spatially correlated channels, irrespective of the channel spatial correlation matrix being full rank or rank-deficient.

\section{B. Effect of Both Temporal and Spatial Correlation on STF Codes}

In these simulations, a typical urban (TU) channel model [22] is considered. The effect of a fading channel with a different spatial and temporal correlation structure on the diversity gain is studied. We assume the fading channel is constant within each OFDM block period, but varies from one OFDM block period to another, according to a first-order Markovian model [16], [17]

$$
\alpha_{i j}^{k}(l)=\varepsilon \alpha_{i j}^{k-1}(l)+\eta_{i j}^{k}(l), \quad 0 \leq l \leq L-1
$$

where the constant $\varepsilon(0 \leq \varepsilon \leq 1)$ determines the amount of the temporal correlation, and $\eta_{i, j}^{k}(l)$ is a zero-mean, complex Gaussian random variable with variance $\sigma_{l} \sqrt{1-\varepsilon^{2}}$. If $\varepsilon=0$, there is no temporal correlation (independent fading), while if $\varepsilon=1$, the channel stays constant over multiple OFDM blocks. We consider three temporal correlation scenarios, $\varepsilon=0, \varepsilon=$ 0.8 , and $\varepsilon=0.95$. The full-diversity STF block codes are obtained by repeating a full-diversity SF block code via (45), described in [8], across $K=1,2,3$ OFDM blocks, respectively. The full-diversity SF block code used for $M_{t}=2$ transmit antennas is constructed from the Alamouti scheme [15] with QPSK modulation via mapping, described in [8]. The OFDM modulation has $N=128$ subcarriers, and the total bandwidth is $4 \mathrm{MHz}$.

First, we consider an STF-coded MIMO-OFDM system without temporal and spatial correlation. The receiver is equipped with $M_{r}=1$ antenna. Fig. 3 depicts the performance results for this scenario. The results show that increasing the

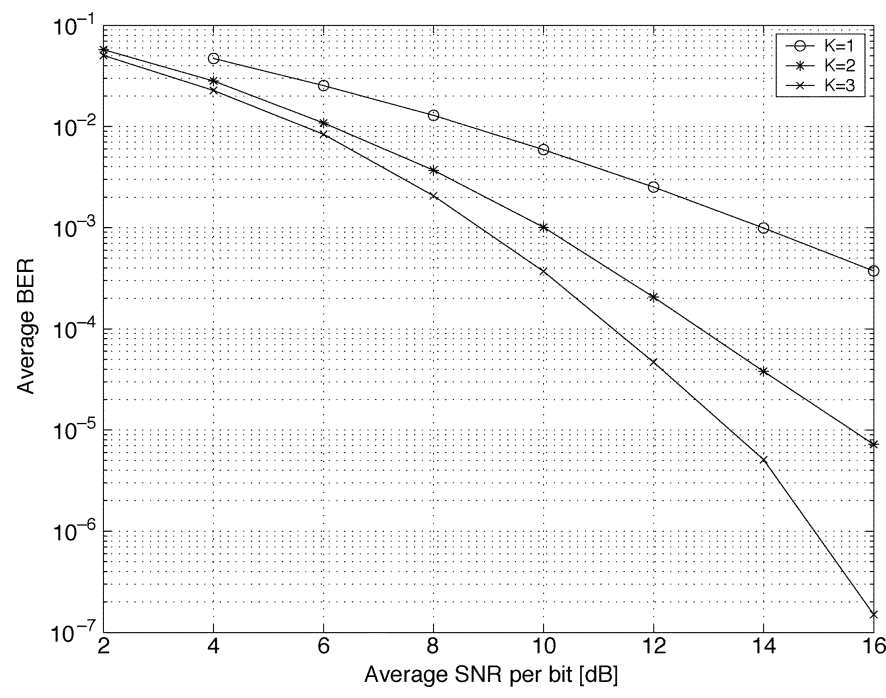

Fig. 3. BER performance of an STF-coded system with $M_{t}=2$ transmit and $M_{r}=1$ receive antennas under independent spatial and temporal fading conditions.

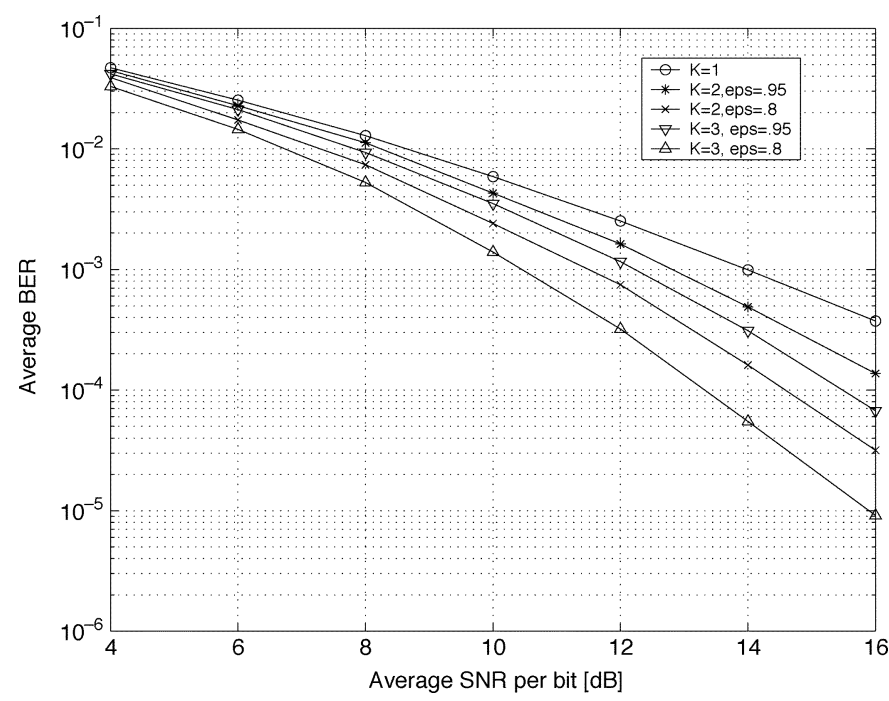

Fig. 4. BER performance of an STF-coded system with $M_{t}=2$ transmit and $M_{r}=1$ receive antennas with independent spatial correlation and different values of $\varepsilon$.

number of jointly encoded OFDM blocks $K$ increases the diversity gain of the system.

Second, we simulated an STF-coded system with temporal correlation only. The receiver has $M_{r}=1$ antenna, and all path gains are spatially independent. Two values, $\varepsilon=0.8$ and $\varepsilon=0.95$, are used to conduct this simulation for $K=1,2,3$. Fig. 4 demonstrates the effect of temporal correlation on the performance curves. It can be seen from this figure that for $\varepsilon=$ 0.95 , the diversity gain achieved is small, compared with the case with $\varepsilon=0.8$. Even when using $K=3$ with $\varepsilon=0.95$, the diversity gain is less than when using $K=2$ with $\varepsilon=0.8$. Thus, the diversity gain when jointly encoding more than one OFDM block decreases with increasing the temporal correlation factor $\varepsilon$. This is intuitive, because for $\varepsilon=0.95$, the channel almost does not change from one OFDM block to another. Hence, there is not much gain in using STF over SF codes in terms of the system diversity order. 


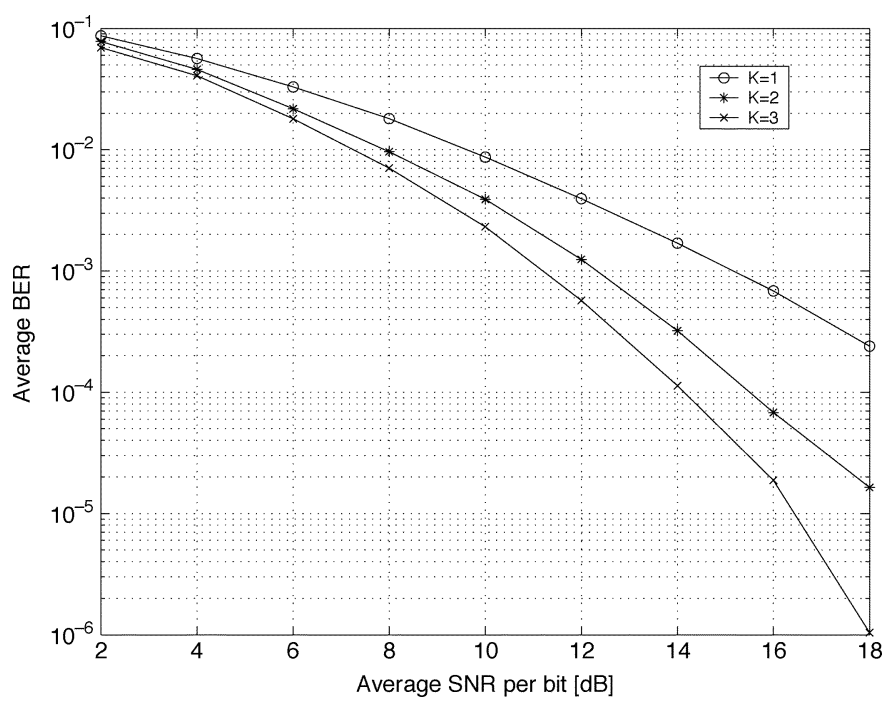

Fig. 5. BER performance of an STF-coded system with $M_{t}=2$ transmit and $M_{r}=1$ receive antennas with spatial correlation and $\varepsilon=0.8$.

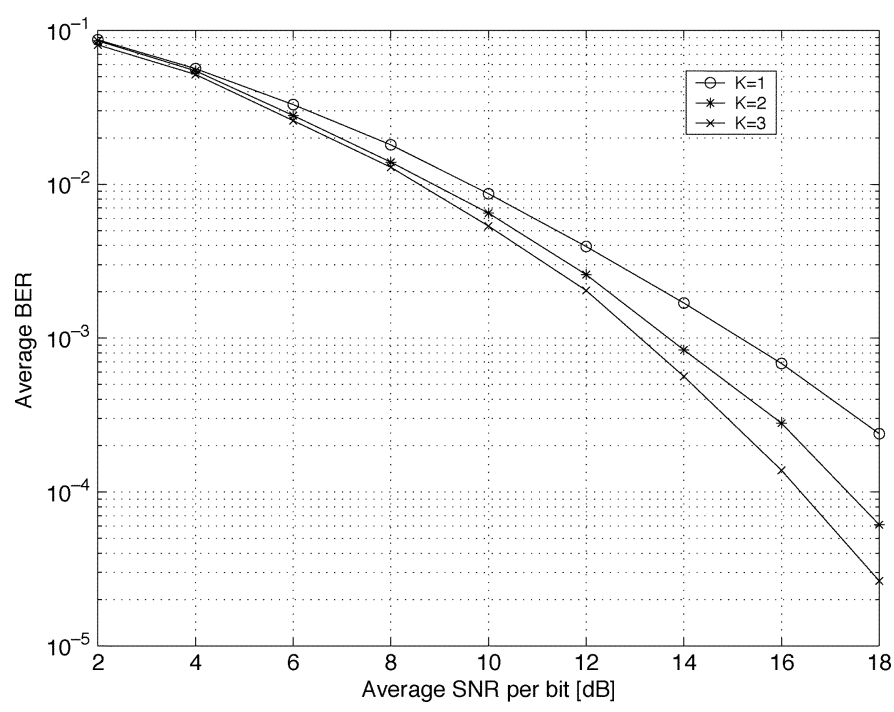

Fig. 6. BER performance of an STF-coded system with $M_{t}=2$ transmit and $M_{r}=1$ receive antennas with spatial correlation and $\varepsilon=0.95$.

Finally, we simulated a system with both temporal and spatial correlation. Figs. 5 and 6 consider a receiver with $M_{r}=1$, the matrix $\mathbf{A}_{l}$ in (55), which determines the spatial correlation is of rank 1, and $\varepsilon$ was 0.8 in Fig. 5 and 0.95 in Fig. 6. It is clear from both figures that the diversity order gained with increasing $K$ is more significant in Fig. 5 with the lower temporal correlation. Figs. 7 and 8 consider a receiver with $M_{r}=2$. In Fig. 7, $\varepsilon$ was 0.8 , and and the matrix $\mathbf{A}_{l}$ is rank 2. It is clear from the figure that increasing $K$ increases the diversity gain achieved. In Fig. 8, $\varepsilon$ was 0.95 , and the matrix $\mathbf{A}_{l}$ is rank 1. As depicted in this figure, increasing $K$ does not have much effect on the diversity gain. This is due to the high temporal correlation $\varepsilon=0.95$, which reduces the diversity gain achieved from the temporal domain.

All the simulation results confirm our intuitive understanding of the factors that determine the diversity order of an STF-coded MIMO-OFDM system. The diversity order or the number of DOFs of a system is given by the multiplication of the DOFs that

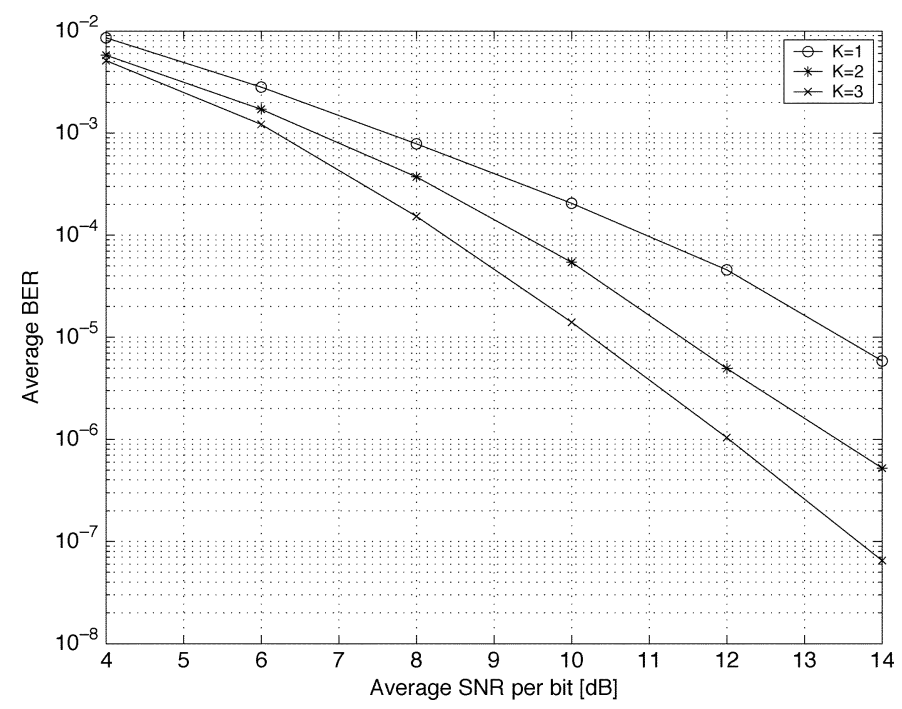

Fig. 7. BER performance of an STF-coded system with $M_{t}=2$ transmit and $M_{r}=2$ receive antennas with spatial correlation and $\varepsilon=0.8$.

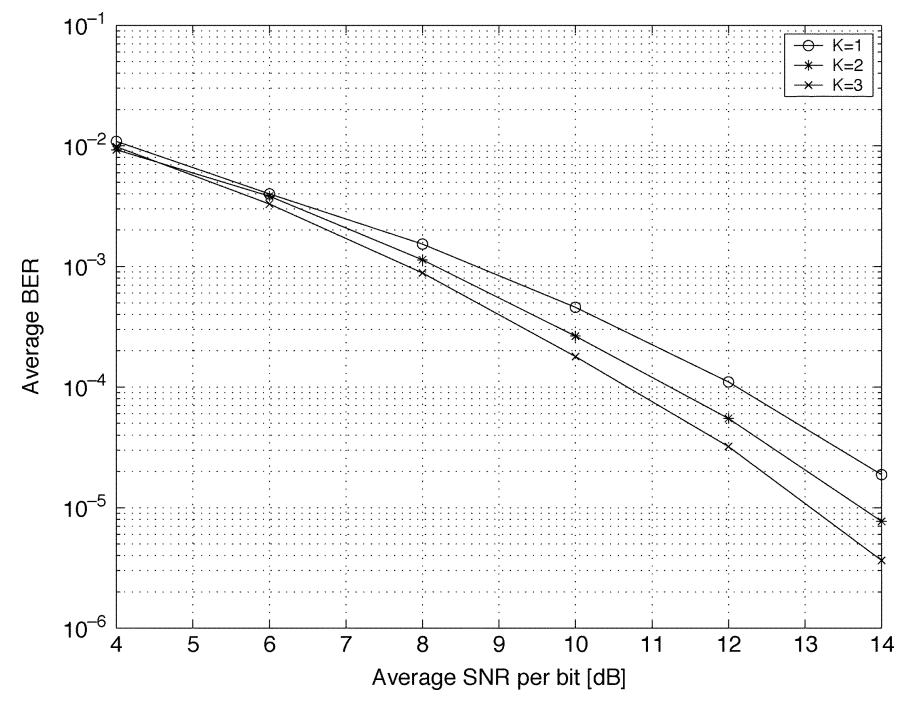

Fig. 8. BER performance of an STF-coded system with $M_{t}=2$ transmit and $M_{r}=2$ receive antennas with spatial correlation and $\varepsilon=0.95$.

we use from each of the three physical domains, time, frequency, and space. This multiplication operation arises due to the independence assumed between the three physical domains. The number of DOFs inside each domain is a function of how much correlation exists between the channels that constitute this domain; for example, the spatial paths between different antenna pairs, different OFDM blocks in the time domain, and different frequency channels in the frequency domain that arise from multipath delays. This function is nothing but the rank of the covariance matrix for the fading process in the associated domain.

\section{CONCLUSION}

In this paper, we analyzed the performance limits of STF-coded MIMO-OFDM systems with a general temporal and spatial correlation. Our analysis handles an arbitrary joint transmit-receive correlation model which covers both the Kronecker and non-Kronecker models. We derived an upper bound on the maximum achievable diversity order of such 
a system. We also showed that the STF code via mapping, designed to achieve full diversity in spatially independent MIMO-OFDM systems, can achieve the full diversity order for channels with arbitrary temporal and spatial fading scenarios. Our analysis framework includes the SF-coded MIMO OFDM system as a special case. For such a system with arbitrary spatial correlation structure, we showed that any SF code designed to achieve full diversity in the independent fading channel can be used to achieve full diversity in an arbitrary spatially correlated channel. Our analytical results for special correlation scenarios are consistent with those existing in the literature. Simulation experiments confirm our theoretical results.

\section{APPENDIX A}

We want to prove that our modeling for the relation between the temporal and spatial fading in (4) is valid for a widely used temporal correlation model. For a first-order Markovian model [16], [17], the fading varies from one OFDM to another, according to

$$
\alpha_{i j}^{k}(l)=\varepsilon \alpha_{i j}^{k-1}(l)+\eta_{i j}^{k}(l), \quad 0 \leq l \leq L-1
$$

where the constant $\varepsilon(0 \leq \varepsilon \leq 1)$ determines the amount of the temporal correlation, and $\eta_{i j}^{k}(l)$ is a zero-mean complex Gaussian random variable with variance $\sigma_{l} \sqrt{1-\varepsilon^{2}}$.

Let $m \geq k$, then for a specific antenna pair $p q$, we have

$$
\alpha_{p q}^{m}(l)=\varepsilon^{m-k} \alpha_{p q}^{k}(l)+\sum_{n=0}^{m-k-1} \varepsilon^{n} \eta_{p q}^{m-n}(l)
$$

Substituting the above equation into $E\left\{\alpha_{i j}^{k}(l) \alpha_{p q}^{m *}(l)\right\}$, we get

$$
\begin{aligned}
E & \left\{\alpha_{i j}^{k}(l) \alpha_{p q}^{m *}(l)\right\} \\
& =E\left\{\alpha_{i j}^{k}(l)\left(\varepsilon^{m-k} \alpha_{p q}^{k *}(l)+\sum_{n=0}^{m-k-1} \varepsilon^{n} \eta_{p q}^{(m-n) *}(l)\right)\right\} \\
& =E\left\{\alpha_{i j}^{k}(l) \varepsilon^{m-k} \alpha_{p q}^{k *}(l)\right\}+0 .
\end{aligned}
$$

Hence, we get

$$
\begin{aligned}
E\left\{\alpha_{i j}^{k}(l) \alpha_{p q}^{m *}(l)\right\} & =\varepsilon^{m-k} E\left\{\alpha_{i j}^{k}(l) \alpha_{p q}^{k *}(l)\right\} \\
& =r_{T}(k-m) E\left\{\alpha_{i j}^{k}(l) \alpha_{p q}^{k *}(l)\right\}
\end{aligned}
$$

\section{REFERENCES}

[1] H. Bölcskei and A. J. Paulraj, "Performance of space-time codes in the presence of spatial fading correlation," in Proc. Asilomar Conf. Signals, Syst., Comput., Oct. 2000, pp. 687-693.

[2] M. Damen, A. Abdi, and M. Kaven, "On the effect of correlated fading on several space-time coding and detection schemes," in Proc. IEEE Veh. Technol. Conf., 2001, pp. 13-16.

[3] V. V. Veeravalli, "On performance analysis for signaling on correlated fading channels," IEEE Trans. Commun., vol. 49, no. 11, pp. 1879, 1883, Nov. 2001.

[4] W. Su, Z. Safar, and K. J. R. Liu, "Diversity analysis of space-time modulation over time-correlated Rayleigh fading channels," IEEE Trans. Inf. Theory, vol. 50, no. 8, pp. 1832-1840, Aug. 2004.
[5] L. J. Cimini, "Analysis and simulation of a digital mobile channel using orthogonal frequency division multiplexing," IEEE Trans. Commun., vol. COM-33, no. 7, pp. 665-675, Jul. 1985.

[6] Y. Li, L. J. Cimini, and N. R. Sollenberger, "Robust channel estimation for OFDM systems with rapid dispersive fading channels," IEEE Trans. Commun., vol. 46, no. 7, pp. 902-915, Jul. 1998.

[7] H. Bölcskei and A. J. Paulraj, "Space-frequency codes for broadband fading channels," in Proc. Int. Symp. Inf. Theory, Washington, DC, Jun. 2001, p. 219.

[8] W. Su, Z. Safar, M. Olfat, and K. J. R. Liu, "Obtaining full-diversity space-frequency codes from space-time code via mapping," IEEE Trans. Signal Process., vol. 51, no. 11, pp. 2905-2916, Nov. 2003.

[9] Z. Liu, Y. Xin, and G. B. Giannakis, "Space-time-frequency coded OFDM over frequency-selective fading channels," IEEE Trans. Signal Process., vol. 50, no. 10, pp. 2465-2476, Oct. 2002.

[10] H. Bölcskei, M. Borgmann, and A. J. Paulraj, "Impact of the propagation environment on the performance of space-frequency coded MIMO-OFDM," IEEE J. Sel. Areas Commun., vol. 21, no. 3, pp. 427-439, Mar. 2003.

[11] E. Bonek, H. Özcelik, M. Herdin, W. Weichselberger, and J. Wallace, "Deficiencies of a popular stochastic MIMO radio channel model," in Proc. Int. Symp. Wireless Pers. Multimedia Commun., Yokosuka, Japan, Oct. 2003, [CD-ROM].

[12] W. Su, Z. Safar, and K. J. R. Liu, "Diversity analysis of space-time-frequency coded broadband OFDM systems," in Proc. 5th Eur. Wireless Conf., Barcelona, Spain, Feb. 2004, [CD-ROM].

[13] _ - "Toward maximum achievable diversity in space, time, and frequency: Performance analysis and code design," IEEE Trans. Wireless Commun., vol. 4, no. 4, pp. 1847-1857, Jul. 2005.

[14] A. K. Sadek, W. Su, and K. J. R. Liu, "Maximum achievable diversity for MIMO-OFDM systems with arbitrary spatial correlation," in Proc. IEEE Global Telecommun. Conf. (GLOBECOM), Dallas, TX, Dec. 2004, pp. 2664-2668

[15] S. Alamouti, "A simple transmit diversity technique for wireless communications," IEEE J. Sel. Areas Commun., vol. 16, no. 8, pp. 1451-1458, Aug. 1998.

[16] H. S. Wang and N. Moayeri, "Finite-state Markov channel-A useful model for radio communication channels," IEEE Trans. Veh. Technol., vol. 44, no. 1, pp. 163-171, Jan. 1996.

[17] H. S. Wang and P.-C. Chang, "On verifying the first-order Markovian assumption for a Rayleigh fading channel model," IEEE Trans. Veh. Technol., vol. 45, no. 2, pp. 353-357, Mar. 1996.

[18] J. G. Proakis, Digital Communications, 3rd ed. New York: McGrawHill, 1995.

[19] A. M. Mathai and S. B. Provost, Quadratic Forms in Random Variables. New York: Marcel Dekker, 1992.

[20] R. A. Horn and C. R. Johnson, Topics in Matrix Analysis. Cambridge, U.K.: Cambridge Univ. Press, 1991.

[21] — Matrix Analysis. Cambridge, U.K.: Cambridge Univ. Press, 1985.

[22] G. Stuber, Principles of Mobile Communication. Norwell, MA: Kluwer, 2001.

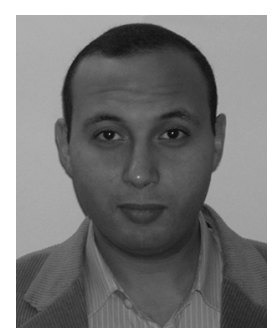

Ahmed K. Sadek (S'03) received the B.S. (with highest honors) and M.S. degrees in electrical engineering from Alexandria University, Alexandria, Egypt, in 2000 and 2003, respectively. He is currently working toward the Ph.D. degree in the Department of Electrical and Computer Engineering, University of Maryland, College Park.

His current research interests are in the area of cooperative communications, wireless and sensor networks, MIMO-OFDM systems, and blind signal processing techniques.

Mr. Sadek's B.S. graduation project won the first award in the IEEE Egypt Section undergraduate student contest in 2000. He also received Graduate School Fellowships in 2003 and 2004 from the University of Maryland. 


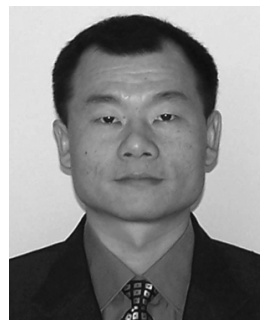

Weifeng Su (M'03) received the Ph.D. degree in electrical engineering from the University of Delaware, Newark, in 2002, and the B.S. and Ph.D. degrees in mathematics from Nankai University, Tianjin, China, in 1994 and 1999, respectively.

He currently is an Assistant Professor with the Department of Electrical Engineering, State University of New York, Buffalo. From June 2002 to March 2005, he was a Postdoctoral Research Associate with the Department of Electrical and Computer Engineering and the Institute for Systems Research (ISR), University of Maryland, College Park. His research interests span a broad range, from signal processing to wireless communications and networking, including space-time coding and modulation for MIMO wireless communications, MIMO-OFDM systems, cooperative communications for wireless networks, and ultra-wideband communications.

Dr. Su received the Signal Processing and Communications Faculty Award from the University of Delaware in 2002 as an outstanding graduate student in the field of signal processing and communications. In 2005, he received the Invention of the Year Award from the University of Maryland. Dr. Su serves as an Associate Editor for the IEEE TRANSACTIONS ON VEHICULAR TECHNOLOGY.

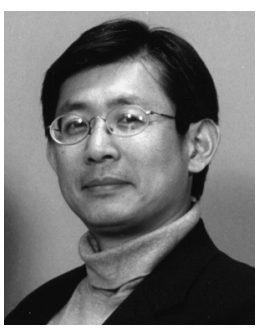

K. J. Ray Liu (F'03) received the B.S. degree from the National Taiwan University, Taipei, in 1983, and the Ph.D. degree from the University of California, Los Angeles, in 1990, both in electrical engineering.

$\mathrm{He}$ is currently a Professor and the Director of the Communications and Signal Processing Laboratories of the Electrical and Computer Engineering Department and Institute for Systems Research, University of Maryland, College Park. His research contributions encompass broad aspects of wireless communications and networking, information forensics and security, multimedia communications and signal processing, bioinformatics and biomedical imaging, and signal processing algorithms and architectures.

Dr. Liu is the recipient of numerous honors and awards, including best paper awards from the IEEE Signal Processing Society twice, the IEEE Vehicular Technology Society, and EURASIP, the EURASIP Meritorious Service Award, and the National Science Foundation Young Investigator Award. He is an IEEE Signal Processing Society Distinguished Lecturer. He also received the Poole and Kent Company Senior Faculty Teaching Award from the A. James Clark School of Engineering, and the Invention of the Year Award, both from the University of Maryland. He is Vice President-Publication and on the Board of Governors of the IEEE Signal Processing Society. He was the Editor-in-Chief of IEEE Signal Processing Magazine, and the founding Editor-in-Chief of the EURASIP Journal on Applied Signal Processing. 